\title{
XXVI. On the general laws of brightness of images
}

\section{Prof. J.D. Everett F.R.S.}

To cite this article: Prof. J.D. Everett F.R.S. (1888) XXVI. On the general laws of brightness of images, Philosophical Magazine Series 5, 25:154, 216-220, DOI: 10.1080/14786448808628174

To link to this article: http://dx.doi.org/10.1080/14786448808628174

曲 Published online: 29 Apr 2009.

Submit your article to this journal $\widetilde{ }$

Џ Article views: 2

Q View related articles $₫$ 


\section{$\left[\begin{array}{lll}{[216} & \text { ] }\end{array}\right.$}

XXVI. On the General Laws of Brightness of Images. By Prof. J. D. Evere'tr, F.R.S., Queen's College, Belfast**

I AM not acquainted with any optical Treatise which con1 tains a general investigation of the brightness of an image seen by rays which have traversed a medium of continuously varying index. The only discussion of the subject that I have met with is contained in chapter xii. of Clausius's 'Mechanical Theory of Heat.' That chapter is entitled " On the Concentration of Rays of Light and Heat," and the main purpose of it is not optical. I have found that its optical results can be obtained by a much simpler method, as set forth in the present paper. The results themselves are probably familiar to leading physicists, but they certainly are not so well known as they deserve to be.

The particular case in which all the refractions and reflexions take place at coaxal spherical surfaces with nearly normal incidence is treated in my edition of 'Deschanel ' (see especially footnote to $\S 768$ of the earlier, or $\S 1037$ of the later, editions), and had been previously treated by a different method in Helmholtz's 'Physiological Optics' (pp. 171-175).

The mode of investigation here adopted has been suggested partly by Clausius's discussion above referred to, partly by an investigation of Kirchhoff's in Poggendorff's Annalen, vol. cix. pp. 284-287, to which Clausius refers, and partly by $\S 334$ (2nd edition) of Thomson and Tait's ' Natural Philosophy.'

We shall begin by establishing the following

Theorem on Apparent Size.

If two small equal plane areas $\mathrm{A}_{1}, \mathrm{~A}_{2}$ are so placed that a ray from the centre of one to the centre of the other is perpendicular to them both, the two solid angles formed by rays at the centre of each which come from the circumference of the other are inversely as the squares of the absolute indices of refraction at the centres at which they are formed. That is, if $\mu_{1} \mu_{2}$ be the indices at the centres of $A_{1} A_{2}, \omega_{1}$ the angle formed at the centre of $A_{1}$ by rays from the circumference of $A_{2}$, and $\omega_{2}$ the solid angle formed at the centre of $\mathrm{A}_{2}$ by rays from the circumference of $A_{1}$, then will

$$
\mu_{1}^{2} A_{1} \omega_{1}=\mu_{2}^{2} A_{2} \omega_{2} \text {. . . . . . . }
$$

As a particular case, if the two indices of refraction are equal, as well as the two areas, the two solid angles will be equal; in other words, the apparent size of the first area as seen from

* Communicated by the Author. 
the second is the same as that of the second as seen from the first.

\section{Proof.}

Let $\mathrm{T}$ denote the time of propagation of light from a point $x y z$ to a point $\xi \eta \xi$, or from the latter point to the former. If $\delta s$ be an indefinitely short line drawn from the point $x y z$ at an inclination $\theta$ to the forward direction of a ray from $\xi \eta \xi$, the increase in $T$ will obviously be the same as for a line $\delta s \cos \theta$ drawn along the ray. Hence, if $v$ denote the velocity of light at $x y z$, and $\mu$ the absolute index of refraction at the same point, we have $d \mathrm{~T}=\frac{1}{v} \delta s \cos \theta$, or

$$
\frac{d \mathrm{~T}}{d s}=\frac{\cos \theta}{v}=\frac{\mu \cos \theta}{\mathrm{V}}, \ldots . .
$$

where $\mathrm{V}$ denotes the velocity in vacuo.

Take the centre of the small area $A_{1}$ as origin of the rectangular coordinates $x y z$, and the centre of $A_{2}$ as origin of $\xi \eta \xi$. Let the axes of $z$ and $\zeta$ be tangential to the ray, and reckoned positive in the outward directions. The axes of $x y$ and of $\xi \eta$ will therefore be in the planes of $A_{1}$ and $A_{2}$ respectively, and we shall choose them so as to satisfy the two conditions

$$
\left[\frac{d^{2} \mathrm{~T}}{d x d \eta}\right]=0, \quad\left[\frac{d^{2} \mathrm{~T}}{d y d \xi}\right]=0, \ldots .
$$

the brackets signifying that the six variables $x, y, z, \xi, \eta, \zeta$ are to be put equal to zero after the performance of the differentiations.

To express the solid angle formed at the centre of $A_{1}$ by rays from the circumference of $A_{2}$, suppose tangents of unit length, forming prolongations of the rays, to be drawn from the centre of $A_{1}$. The solid angle (being small) will be numerically equal to the base of the cone or pyramid enclosed by the tangents, or to the projection of this base on the plane of $x y$. Let $x^{\prime}$ and $y^{\prime}$ be the coordinates of the projection of the extremity of one of these unit-tangents, then the solid angle will be equal to the area traced by the point $x^{\prime} y^{\prime}$ in the plane of $A_{1}$, while the point $\xi \eta$, from which the ray proceeds, moves round the circumference of $\mathrm{A}_{2}$. The coordinates $x^{1}$ and $y^{\prime}$ are obviously equal to the cosines of the angles which the ray makes with the axes of $x$ and $y$.

Hence, putting $\cos \theta$ in (2) successively equal to $x^{\prime}$ and $y^{\prime}$, we have

$$
x^{\prime}=\frac{\mathrm{V}}{\mu_{1}} \frac{d \mathrm{~T}}{d x}, \quad y^{\prime}=\frac{\mathrm{V}}{\mu_{1}} \frac{d \mathrm{~T}}{d y}, \quad \ldots . .
$$

$\mu_{1}$ denoting the value of $\mu$ at the centre of $A_{1}$. 
Again, since $x^{\prime}$ and $y^{\prime}$ are functions of the two independent variables $\xi$ and $\eta$, and vanish with them, we have, to the first order of small quantities,

But by (4),

$$
x^{\prime}=\left[\frac{d x^{\prime}}{d \xi}\right] \xi+\left[\frac{d x^{\prime}}{d \eta}\right] \eta, \quad y^{\prime}=\left[\frac{d y^{\prime}}{d \xi}\right] \xi+\left[\frac{d y^{\prime}}{d \eta}\right] \eta .
$$

$$
\frac{d x^{\prime}}{d \xi}=\frac{\mathrm{V}}{\mu_{1}} \frac{d^{2} \mathrm{~T}}{d x d \xi^{\prime}} \quad \frac{d x^{\prime}}{d \eta}=\frac{\mathrm{V}}{\mu_{1}} \frac{d^{2} \mathrm{~T}}{d x d \eta}, \quad . \quad .
$$

Thus equations (5) reduce, by the help of (3), to

$$
x^{\prime}=\frac{\mathrm{V}}{\mu_{1}}\left[\frac{d^{2} \mathrm{~T}}{d x d \xi}\right] \xi, \quad y^{\prime}=\frac{\mathrm{V}}{\mu_{1}}\left[\frac{d^{2} \mathrm{~T}}{d y d \eta}\right] \eta . .
$$

Hence $x^{\prime}$ has a constant ratio to $\xi$, and $y^{\prime}$ a constant ratio to $\eta$. The product of these two ratios will be the ratio of the area traced by the point $x^{\prime} y^{\prime}$ to the area traced by $\xi \eta$, that is, it will be the ratio of the solid angle $\omega_{1}$ to the area $A_{2}$. We have accordingly

$$
\omega_{1}=\left(\frac{\mathrm{V}}{\mu_{1}}\right)^{2}\left[\frac{d^{2} \mathrm{~T}}{d x d \xi}\right]\left[\frac{d^{2} \mathrm{~T}}{d y d \eta}\right] \mathrm{A}_{2} \cdot \ldots . .
$$

By applying similar reasoning to rays from the perimeter of $A_{1}$ to the centre of $A_{2}$, we shall find (with similar notation)

$$
\begin{gathered}
\xi^{\prime}=\frac{\mathrm{V}}{\mu_{2}}\left[\frac{d^{2} \mathrm{~T}}{d x d \xi}\right] x, \quad \eta^{\prime}=\frac{\mathrm{V}}{\mu_{2}}\left[\frac{d^{2} \mathrm{~T}}{d y d \eta}\right] y, \quad . \quad . \quad . \\
\omega_{2}=\left(\frac{\mathrm{V}}{\mu_{2}}\right)^{2}\left[\frac{d^{2} \mathrm{~T}}{d x d \xi}\right]\left[\frac{d^{2} \mathrm{~T}}{d y d \eta}\right] \mathrm{A}_{1} . \quad . \quad . .
\end{gathered}
$$

By comparison of (8) and (10) we have

$$
\mu_{1}^{2} \mathrm{~A}_{1} \omega_{1}=\mu_{2}^{2} \mathrm{~A}_{2} \omega_{2} \text {. . . . . . . . }
$$

The rays considered in the foregoing proof may undergo any amount of either gradual or abrupt bending by refraction, and may be reflected any number of times; but there must be no abrapt difference between the histories of rays which come from consecutive points.

We now proceed to apply this theorem to the investigation of brightness.

The brightness of an object as seen from any point is measured by

$$
\frac{q}{\mathrm{~A} \omega}, \cdot \text {. . . . . . }
$$

A denoting a small area at the point, sensibly perpendicular to the rays which reach it from the object, $\omega$ a small solid angle of arbitrary magnitude, and $q$ the quantity of light from the object which converges to $A$ (and also diverges 
from $A$ if not stopped) in pencils of magnitude $\omega$. The justness of this measure is obvious from the fact that if we halve either $A$ or $\omega$ we shall halve the quantity of light that will come into consideration.

When the small area $A$ is taken on the surface of the object itself, the value of the expression (11) is called the intrinsic brightness of the object.

In the case of the two small areas which we have been discussing, let $q$ denote the quantity of light sent by $A_{2}$ on the road to $A_{1}$. If none of it is lost on the road, it all reaches $A_{1}$; thus the same quantity of light which leaves $A_{2}$ in pencils of angle $\omega_{2}$ reaches $A_{1}$ in pencils of angle $\omega_{1}$. Let $I_{2}$ denote the intrinsic brightness of $A_{2}$, and $I$ its apparent brightness as seen from $A_{1}$; then

$$
\mathrm{I}=\frac{q}{\mathrm{~A}_{1} \omega_{1}}=\frac{\mathrm{A}_{2} \omega_{2}}{\mathrm{~A}_{1} \omega_{1}} \frac{q}{\mathrm{~A}_{2} \omega_{2}}=\left(\frac{\mu_{1}}{\mu_{2}}\right)^{2} \frac{q}{\mathrm{~A}_{2} \omega_{2}}=\left(\frac{\mu_{1}}{\mu_{2}}\right)^{2} \mathrm{I}_{2} .
$$

By supposing $\mu_{2}$ equal to $\mu_{1}$ we obtain the following theorem. If the eye and the object are in media of the same index, and no light is stopped on its way from the object to the eye, the apparent brightness is equal to the intrinsic brightness, notwithstanding any refractions or total reflewions that the rays may have undergone between the object and the eye.

If light is lost on the way, the above expression for the apparent brightness must be multiplied by a coefficient $k$ less than unity, which, by well-established laws of Optics, is the same for rays going from $A_{2}$ to $A_{1}$ as for those going from $A_{1}$ to $A_{2}$ (for the same kind of light). If $I_{2}$ and $I_{1}$ are the intrinsic brightnesses of the two areas, their apparent brightnesses when each is seen from the other will be

$$
k\left(\frac{\mu_{1}}{\mu_{2}}\right) I_{2}, \text { and } k\left(\frac{\mu_{2}}{\mu_{1}}\right)^{2} I_{1} \text {. . . . . }
$$

When an observer sees the area $A_{2}$, the pupil of his eye may be taken as the area $A_{1}$; the foregoing computations of apparent brightness accordingly assume that all parts of the pupil receive rays from every part of $A_{2}$. If part of the pupil be covered, the apparent brightness of the object will be diminished; and a similar diminution will occur when the rays from the object are collected into a beam of such small section as not to fill the pupil. This is the cause of the falling off of light which is observed in the use of high magnifying-powers with optical instruments.

In the case of light sent out obliquely from the surface of an object, the above investigations will remain applicable if 
we understand by $A_{2}$ the projection of this surface on a normal section of the beam.

When $A_{1}$ is the image of $A_{2}$ the "theorem on apparent size" requires modification, for the centre of $A_{1}$ will no longer receive one ray from each point of $A_{2}$, but will receive all its rays from one point only, which we shall regard as the centre of $A_{2}$. Let a section orthogonal to all the rays which go from the centre of $A_{2}$ to the centre of $A_{1}$ be taken anywhere in the intermediate region, but not so as to coincide with a surface at which there is an abrupt change of direction in any of the rays. For instance, if the image is formed by a lens, the section may be taken either in the substance of the lens or in the external medium, but must not coincide with a face of the lens. Divide this section into parts comparable in size with $A_{1}$ or $A_{2}$, and let $A_{3}$ denote the area of one of these parts. Also let $\mu_{3}$ denote the index of refraction at the centre of $A_{3}$. Since $A_{1}$ is the image of $A_{2}$, the rays which go from the perimeter of $A_{2}$ to a fixed point of $A_{3}$ will go on to the perimeter of $A_{1}$; thus $A_{2}$ and $A_{1}$ will subtend the same solid angle as seen from $A_{3}$, call it $\omega_{3}$. Hence, by applying (1) first to $A_{1}$ and $A_{3}$ and then to $A_{3}$ and $A_{2}$, we have

$$
\mu_{1}^{2} A_{1} \omega_{1}=\mu_{3}^{2} A_{3} \omega_{3}=\mu_{2}^{2} A_{2} \omega_{2} \text {, . . . }
$$

$\omega_{1}$ and $\omega_{2}$ denoting the solid angles formed at $A_{1}$ and $A_{2}$ by rays which traverse the perimeter of $A_{3}$.

Equations (14) show that the ratio of $\omega_{1}$ to $\omega_{2}$ is the same for all the portions such as $A_{3}$ into which the intermediate section is divided. Let $\Omega_{1} \Omega_{2}$ be the whole solid angles at $A_{1} A_{2}$ formed by the aggregate of all the rays which go from $A_{2}$ to $A_{1} . \quad \Omega_{1}$ is the sum of all the partial angles $\omega_{1}$, and $\Omega_{2}$ is the sum of all the partial angles $\omega_{2}$; hence we have

$$
\mu_{1}^{2} \mathrm{~A}_{1} \Omega_{1}=\mu_{2}^{2} \mathrm{~A}_{2} \Omega_{2}, \text {. . . . . }
$$

a result which is similar in form to (1).

The rays which go from $A_{2}$ to $A_{1}$ diverge from $A_{2}$ in the solid angle $\Omega_{2}$, and converge to $A_{1}$ in the solid angle $\Omega_{1}$. Let $Q$ be the whole light sent from $A_{2}$ on the way to $\AA_{1}$, and $k \mathrm{Q}$ the portion of it which actually reaches $\hat{A}_{1}$, then the intrinsic brightness $I$ of the real image $A_{1}$ will be

$$
\mathrm{I}=\frac{k \mathrm{Q}}{\mathrm{A}_{1} \Omega_{1}}=\frac{k \mathrm{~A}_{2} \Omega_{2}}{\mathrm{~A}_{1} \Omega_{1}} \frac{\mathrm{Q}}{\mathrm{A}_{2} \Omega_{2}}=k\left(\frac{\mu_{1}}{\mu_{2}}\right)^{2} \frac{\mathrm{Q}}{\mathrm{A}_{2} \Omega_{2}}=k\left(\frac{\mu_{1}}{\mu_{2}}\right)^{2} \mathrm{I}_{2}
$$

as in (13). When the pupil of the eye is placed at the image, the brightness of the field of view will be identical with this intrinsic brightness of the image, if the pupil is filled. The apparent brightness of $A_{2}$ as seen from $A_{1}$ will then be unaffected by the circumstance that $A_{1}$ is the image of $A_{2}$. 